\title{
A CH-type Inequality For Real Experiments
}

\author{
A. Shafiee* \\ 1)Department of Chemistry, Kashan University, \\ Kashan, 87317-51167, Iran. \\ 2)Institute for Studies in Theoretical Physics \& Mathematics, \\ P.O.Box 19395-5531, Tehran, Iran.
}

June 19, 2018

\begin{abstract}
We derive an efficient CH-type inequality. Quantum mechanics violates our proposed inequality independent of the detection-efficiency problem.
\end{abstract}

In photonic Bell-type experiments [1], when photon pairs with parallel linear polarizations are emitted, one can consider a Clauser-Horne $(\mathrm{CH})$ inequality [2], at the level of hidden variables, in the form

$$
-1 \leq S_{r q, H V}\left(\widehat{a}, \widehat{b}, \widehat{a}^{\prime}, \widehat{b^{\prime}}, \lambda\right) \leq 0
$$

where

$$
\begin{aligned}
S_{r q, H V}\left(\widehat{a}, \widehat{b}, \widehat{a^{\prime}}, \widehat{b^{\prime}}, \lambda\right)= & p_{r}^{(1)}(\widehat{a}, \lambda)\left[p_{q}^{(2)}(\widehat{b}, \lambda)-p_{q}^{(2)}\left(\widehat{b}^{\prime}, \lambda\right)\right] \\
& +p_{r}^{(1)}\left(\widehat{a^{\prime}}, \lambda\right)\left[p_{q}^{(2)}(\widehat{b}, \lambda)+p_{q}^{(2)}\left(\widehat{b}^{\prime}, \lambda\right)\right] \\
& -p_{r}^{(1)}\left(\widehat{a^{\prime}}, \lambda\right)-p_{q}^{(2)}(\widehat{b}, \lambda)
\end{aligned}
$$

In (2), we are considering four sub-ensemble of photon pairs with linear polarizations along $(\widehat{a}, \widehat{b}),\left(\widehat{a}, \widehat{b^{\prime}}\right),\left(\widehat{a^{\prime}}, \widehat{b}\right)$, and $\left(\widehat{a}^{\prime}, \widehat{b}^{\prime}\right)$ in which one registers

*E-mail: shafiee@theory.ipm.ac.ir 
the result $r$ for the first photon with an appropriate probability $p_{r}^{(1)}$ and the result $q$ for the second one with probability $p_{q}^{(2)}$ where $r, q= \pm 1$.

To extend the $\mathrm{CH}$ inequality to a more efficient one, we propose a function $S_{r q}^{\prime}$ in the form:

$$
\begin{aligned}
S_{r q, H V}^{\prime}\left(\widehat{a}, \widehat{b}, \widehat{a^{\prime}}, \widehat{b^{\prime}}, \lambda\right)= & p_{r}^{(1)}(\widehat{a}, \lambda)\left[p_{q}^{(2)}(\widehat{b}, \lambda)-p_{q}^{(2)}\left(\widehat{b^{\prime}}, \lambda\right)\right] \\
& +p_{r}^{(1)}\left(\widehat{a^{\prime}}, \lambda\right)\left[p_{q}^{(2)}(\widehat{b}, \lambda)+p_{q}^{(2)}\left(\widehat{b^{\prime}}, \lambda\right)\right] \\
& -p_{r}^{(1)}\left(\widehat{a^{\prime}}, \lambda\right) p_{r}^{(2)}\left(\widehat{a^{\prime}}, \lambda\right)-p_{q}^{(1)}(\widehat{b}, \lambda) p_{q}^{(2)}(\widehat{b}, \lambda)
\end{aligned}
$$

In contrast to the $\mathrm{CH}$ inequality, the upper limit of relation (3) should not necessarily be equal to zero in non ideal experiments. To avoid this difficulty, we first consider the following inequality for the single-particle probabilities in an actual experiment:

$$
0 \leq p_{j}^{(k)}\left(\widehat{x}_{k}, \lambda\right) \leq 1-p_{0}^{(k)}\left(\widehat{x}_{k}, \lambda\right)
$$

where $\widehat{x}_{1}=\widehat{a}, \widehat{a^{\prime}}$ or $\widehat{b}, \widehat{x}_{2}=\widehat{b}, \widehat{b^{\prime}}$ or $\widehat{a^{\prime}}$ and $j= \pm 1$. The function $p_{0}^{(k)}\left(\widehat{x}_{k}, \lambda\right)$ denotes non-detection probability for the $k$ th photon with the polarization along $\widehat{x}_{k}$. Then, we define the following relation:

$$
\sum_{j= \pm 1} p_{j}^{(k)}\left(\widehat{x}_{k}, \lambda\right)=\alpha^{(k)}\left(\widehat{x}_{k}, \lambda\right)=1-p_{0}^{(k)}\left(\widehat{x}_{k}, \lambda\right)
$$

where $\alpha^{(k)}\left(\widehat{x}_{k}, \lambda\right)$ is a measure of inefficiencies at the level of hidden-variables. For more convenience, we call $\alpha^{(1)}(\widehat{a}, \lambda) \equiv \alpha_{1}, \alpha^{(1)}\left(\widehat{a^{\prime}}, \lambda\right) \equiv \alpha_{1}^{\prime}, \alpha^{(2)}\left(\widehat{a^{\prime}}, \lambda\right) \equiv$ $\alpha_{2}^{\prime}, \alpha^{(2)}(\widehat{b}, \lambda) \equiv \beta_{2}, \alpha^{(2)}\left(\widehat{b^{\prime}}, \lambda\right) \equiv \beta_{2}^{\prime}$ and $\alpha^{(1)}(\widehat{b}, \lambda) \equiv \beta_{1}$. According to the definition of the inefficiency measures in $(5)$, we have:

$$
\begin{aligned}
\int_{\Lambda} \alpha^{(1)}\left(\widehat{x}_{1}, \lambda\right) \alpha^{(2)}\left(\widehat{x}_{2}, \lambda\right) \rho(\lambda) d \lambda & =\int_{\Lambda} \sum_{r, q= \pm 1} p_{r}^{(1)}\left(\widehat{x}_{1}, \lambda\right) p_{q}^{(2)}\left(\widehat{x}_{2}, \lambda\right) \rho(\lambda) d \lambda \\
& =\sum_{r, q} P_{r q}^{(12)}\left(\widehat{x}_{1}, \widehat{x}_{2}\right) \equiv M\left(\widehat{x}_{1}, \widehat{x}_{2}\right)
\end{aligned}
$$

where $P_{r q}^{(12)}\left(\widehat{x}_{1}, \widehat{x}_{2}\right)$ is the joint probability for getting the results $r$ and $q$ for the first and second photons along $\widehat{x}_{1}$ and $\widehat{x}_{2}$, respectively, at the experimental level and $\rho(\lambda)$ is a probability density in space $\Lambda$. One can easily show that $M\left(\widehat{x}_{1}, \widehat{x}_{2}\right)=1-P_{0}^{(1)}\left(\widehat{x}_{1}\right)-P_{0}^{(2)}\left(\widehat{x}_{2}\right)-P_{00}^{(12)}\left(\widehat{x}_{1}, \widehat{x}_{2}\right)$ which is a measure of 
non-detection probabilities in real experiments. Now, we make the following assumption:

A- The experimental probabilities of non-detection are independent of the polarization directions.

It is important to notice that we are suggesting $\mathbf{A}$ only at the observational level. This indicates that $M\left(\widehat{x}_{1}, \widehat{x}_{2}\right)$ should be independent of any direction. Using $\mathbf{A}$ and multiplying the limits of $S_{r q, H V}^{\prime}$ in (3) through $\rho(\lambda)$ and integrating over the space $\Lambda$, we get the following inequality at the experimental level:

$$
-1 \leq S_{r q, \exp }^{\prime}\left(\widehat{a}, \widehat{b}, \widehat{a^{\prime}}, \widehat{b^{\prime}}\right) \leq 0
$$

This is our extended CH inequality where $S_{r q, \exp }^{\prime}$ is defined as follows:

$$
\begin{aligned}
S_{r q, \exp }^{\prime}\left(\widehat{a}, \widehat{b}, \widehat{a^{\prime}}, \widehat{b^{\prime}}\right)= & P_{r q}^{(12)}(\widehat{a}, \widehat{b})-P_{r q}^{(12)}\left(\widehat{a}, \widehat{b^{\prime}}\right)+P_{r q}^{(12)}\left(\widehat{a^{\prime}}, \widehat{b}\right)+P_{r q}^{(12)}\left(\widehat{a^{\prime}}, \widehat{b^{\prime}}\right) \\
& -P_{r r}^{(12)}\left(\widehat{a^{\prime}}, \widehat{a^{\prime}}\right)-P_{q q}^{(12)}(\widehat{b}, \widehat{b})
\end{aligned}
$$

In deriving (7), we have used Bell's locality (factorizability) assumption [3]. The inequality (7) is violated by quantum mechanical predictions in nonideal regime. To show this one can define the joint probability $P_{++, Q M}^{(12)}(\widehat{a}, \widehat{b})$ in a real experiment as [4]

$$
P_{++, Q M}^{(12)}(\widehat{a}, \widehat{b}) \approx \frac{1}{4} \eta_{1} \eta_{2} f[1+F \cos 2(\widehat{a}-\widehat{b})]
$$

where $\eta_{k}(k=1,2)$ and $f$ are respectively the efficiencies of the detectors and the collimators, and $F$ is a measure of the correlation of the two photons. The efficiency parameters in (9) are usually believed to be independent of the polarization directions in the literature. So, the assumption $\mathbf{A}$ is naturally honored in quantum mechanical calculations.

Now, we consider the case $|\widehat{a}-\widehat{b}|=\left|\widehat{a^{\prime}}-\widehat{b}\right|=\left|\widehat{a^{\prime}}-\widehat{b^{\prime}}\right|=\frac{\varphi}{2}$ and $\left|\widehat{a}-\widehat{b^{\prime}}\right|=\frac{3 \varphi}{2}$. Substituting (9) and relations similar to it in (8) and choosing $r=q=+1$, we get

$$
S_{++, Q M}^{\prime}(\varphi) \approx \frac{1}{4} \eta_{1} \eta_{2} f F[3 \cos \varphi-\cos 3 \varphi-2]
$$

Considering the upper limit in (7), we get 


$$
(3 \cos \varphi-\cos 3 \varphi) \leq 2
$$

This is violated for certain ranges of $\varphi$. We note that none of the efficiency parameters appear in this inequality. Thus, quantum mechanics violates (7) independent of the efficiencies of the apparatuses.

\section{References}

[1] W. Tittle and G. Weihs, Quantum Information and Computation, 1, 3 (2001).

[2] J. F. Clauser and M. A. Horne, Phys. Rev. D 10, 526 (1974).

[3] A. Shimony, in Sixty-Two Years of Uncertainty: Historical, Philosophical, and Physical Inquiries into the Foundations of Quantum Mechanics, edited by A. Miller (Plenum, New York, 1990), pp. 33-43.

[4] . F. Clauser and A. Shimony, Rep. Prog. Phys., 41, 1881 (1978). 